\title{
Serotipos de Salmonella identificados en los servicios de salud de México
}

\author{
Lucina Gutiérrez-C ogco, Q .B.P., ${ }^{(1)}$ Edith Montiel-Vázquez, Q .F.B., ${ }^{(1)}$ \\ Pablo Aguilera-Pérez, Q .F.B., ${ }^{(1)}$ María del C armen G onzález-Andrade, M.C. .(2)
}

\begin{abstract}
Gutiérrez-Cogco L, Montiel-Vázquez E, Aguilera-Pérez P, González-Andrade MC.

Serotipos de Salmonella identificados en los servicios de salud de México. Salud Publica Mex 2000;42:490-495.
\end{abstract}

\section{Resumen}

Objetivo. Dar a conocer las variaciones de los serotipos de cepas de Salmonella aislados en diversos laboratorios públicos y privados de la República mexicana, así como en el del Instituto de Diagnóstico y Referencia Epidemiológicos (InDRE). Material y métodos. Se analizaron los serotipos de 24394 cepas de Salmonella aisladas de diversas fuentes por los laboratorios de salud pública y privados de México, entre 1972 y 1999, 15843 (64.9\%) de origen humano y $8551(35.1 \%)$ de origen no humano siguiendo el esquema de Kauffmann-W hite y utilizando antisueros producidos en el InDRE, de acuerdo con los lineamientos del Centro de Control y Prevención de Enfermedades, A tlanta (GA), de los Estados Unidos de América. Resultados Se identificaron 199 serotipos, el más frecuente en muestras clínicas fue S. Typhimurium (20.4\%) y, en segundo lugar, S. Enteritidis (18.3\%). En este trabajo se muestra como S. Enteritidis tuvo un aumento gradual y desplazó a S. Typhimurium en los aislamientos desde 1991, y a la fecha es el serotipo que más se aísla. En cambio, en muestras no humanas el serotipo aislado con mayor frecuencia fue S. Derby $(13.8 \%)$, seguido por S. A natum (8.5\%). Conclusiones Los serotipos más frecuentes en México, tanto en muestras humanas como no humanas son: S. Typhimurium, S. Enteritidis, S. D erby, S. Agona y S. A natum. D esde el punto de vista epidemiológico es necesario conocer cuáles son los serotipos circulantes y los de nueva introducción para poder determinar las acciones de prevención requeridas para los casos.

Palabras clave: Salmonella; serotipificación; México

\author{
Gutiérrez-Cogco L, Montiel-Vázquez E, \\ Aguilera-Pérez P, González-Andrade MC. \\ Salmonella serotypes isolated \\ in Mexico's health services. \\ Salud Publica Mex 2000;42:490-495.
}

\begin{abstract}
A bstract
Objective.To identify the different Salmonella strain serotypes isolated at public and private laboratories in Mexico and at the Institute for Epidemiologic D iagnosis and Referral (InDRE). Material and methods. A total of 24394 Salmonella strains collected from 1972 to 1999 in public and private health laboratories of Mexico were analyzed with the Kauffmann-W hite method, using antisera produced by InDRE, according to Centers for $D$ isease Control and Prevention (CDC, Atlanta, GA) standards; 15843 $(64.9 \%)$ samples were from human sources and 8551 (35.1\%) from non-human sources. Results. 0 ne hundred ninety nine different serotypes were identified. The most frequent serotype in human beings was $\mathrm{S}$. Typhimurium (20.4\%), followed by S. Enteritidis (18.3\%). In the past few years, the frequency of $S$. Enteritidis has been increasing, surpassing that of S. Typhimurium since 1991. Presently S. Enteritidis is the most frequently isolated serotype. In nonhuman sources, S. Derby (13.8\%) and S. A natum (8.5\%) are the most frequent strains. Conclusions Salmonella serotypes most frequently isolated in Mexico are: $\mathbf{S}$. Typhimurium, S. Enteritidis, S. D erby, S. A gona y S. A natum. From the epidemiologic standpoint, it is necessary to identify circulating and emerging Salmonella serotypes in order to target pertinent preventative interventions.
\end{abstract}

Keywords: Salmonella; serotyping; Mexico

(1) Laboratorio de Bacteriología Entérica, Departamento de Bacteriología, Instituto de Diagnóstico y Referencia Epidemiológicos, México.

(2) Dirección General de Epidemiología, Secretaría de Salud, México.

Fecha de recibido: 15 de marzo de 2000 - Fecha de aprobado: 22 de agosto de 2000 Solicitud de sobretiros: Q.B.P. Lucina Gutiérrez Cogco. Departamento de Bacteriología, Instituto de Diagnóstico y Refencia Epidemiológicos. Carpio 470, colonia Santo Tomás, D elegación Miguel Hidalgo, 11340 México, D.F. Correo electrónico: Igutierrez@ mail.ssa.gob.mx 
L a salmonelosis es una infección de importancia en salud pública debido al impacto socioeconómico que ocasiona tanto en los países en desarrollo como en los desarrollados. Es una enfermedad transmitida por los alimentos los cuales causan la mayor parte de los brotes que afectan a centenares de personas $y$, aunque puede ser causada por cualquiera de los casi 2500 serotipos que existen hasta hoy, los que se aíslan con mayor frecuencia en México son Salmonella Enteritidis y $S$. Typhimurium. ${ }^{1,2}$ Es una enfermedad aguda de distribución mundial, con variaciones en la frecuencia de serotipos de un país a otro, con importancia en áreas que no han alcanzado las condiciones de saneamiento e higiene adecuados y no cuentan con medidas de salud pública óptimas. Afecta a todos los grupos de edad, con mayor incidencia en los extremos de la vida: en menores de cinco años y mayores de 60 años de edad, que son los grupos más vulnerables. ${ }^{3,4}$ Tiene una incidencia estacional, por lo que el canal endémico registra aumento de casos a partir del mes de mayo, con pico máximo en julio y agosto y una declinación a partir de septiembre. ${ }^{5,6}$

Desde la década de los ochenta, la incidencia de salmonelosis de origen alimentario ha aumentado considerablemente en el mundo industrializado y ha alcanzado proporciones epidémicas en varios países. ${ }^{7,8}$ Este incremento es el resultado de una combinación de factores relacionados con el desarrollo en la industrialización en todas las fases de producción de alimentos, cambios en la práctica del manejo de los alimentos, así como el almacenamiento, distribución y preparación de los mismos. Estos cambios han tenido como consecuencia nuevos problemas en la higiene de los alimentos al originar una fácil diseminación de Salmonella así como de otros gérmenes patógenos en los alimentos. ${ }^{9}$

En México, en los últimos cinco años (1994 a 1998), las notificaciones de casos por salmonelosis registran un incremento de 100342 casos, en 1994, a 215 155, en 1998 (tasa de 111.21 y 223.53 por 100000 habitantes, respectivamente), con una mayor incidencia en los grupos de 15 a 24 años, de 25 a 44 años y de 45 a 64 años, siendo el segundo el grupo más afectado. En cuanto a frecuencia con relación a los meses del año ésta se intensifica a partir de los meses de abril y mayo alcanzando un pico en julio, con una disminución en septiembre y octubre. Los estados más afectados han sido Tabasco, Coahuila, Chiapas y Quintana Roo. ${ }^{10}$

Desde 1940, en el Instituto de Diagnóstico y Referencia Epidemiológicos (InDRE) se realiza la serotipificación de salmonelas aisladas de muestras clínicas, ambientales y de alimentos. Entre 1940 y 1960, Olarte y Varela encontraron 74 serotipos diferentes. ${ }^{11}$
A partir de 1972, y debido al brote de tifoidea que ocurrió en la parte central de la República mexicana, se empezaron a serotipificar las salmonelas en forma continua y en un estudio similar, llevado a cabo entre 1974 y 1981, se encontraron 80 serotipos diferentes, 35 de los cuales no se habían reportado en años anteriores. ${ }^{2}$

El presente informe es una revisión de 31 años, de 1972 a 1999, para dar a conocer las variaciones de los serotipos de cepas enviadas por diferentes laboratorios de toda la República mexicana durante este periodo.

\section{Material y métodos}

Se analizaron 24394 cepas de Salmonella aisladas en México durante el periodo de 1972 a 1999. Del total, $1372(5.6 \%)$ cepas de muestras clínicas fueron aisladas en el Laboratorio de Bacteriología Entérica del InDRE y el resto, de alimentos y ambientales, en los 30 laboratorios estatales de salud pública de la Secretaría de Salud, así como de la industria privada. Las cepas puras fueron enviadas por los laboratorios para serotipificación en el InDRE. El aislamiento e identificación se llevó a cabo de acuerdo con el Manual de diagnóstico de infecciones gastrointestinales* y la serotipificación de acuerdo con el esquema de Kauffmann-White, ${ }^{12}$ utilizando sueros somáticos y flagelares producidos por el Laboratorio de Producción de Sueros del propio InDRE. Los medios de cultivo utilizados se prepararon de acuerdo con el marbete del fabricante de cada uno y se realizaron pruebas de control de calidad utilizando dos cepas de referencia específicas para cada uno de los medios, una para prueba positiva y otra para prueba negativa, los antisueros se probaron con bacterias del mismo antígeno somático y con otros que pudieran dar reacciones cruzadas y, en caso de éstas, se procedía a adsorber el antisuero con el antígeno somático correspondiente.

\section{Resultados}

De las 24394 cepas de Salmonella, 15843 (64.9\%) fueron de origen humano y 8551 (35.1\%) de origen no humano. Para conocer mejor la distribución de los serotipos se dividieron en varios grupos dependiendo de la fuente de aislamiento. Del grupo humano, $95.2 \%$ fueron de coprocultivo y $4.8 \%$ de origen extraintesti-

* Instituto Nacional de Diagnóstico y Referencia Epidemiológicos. Diagnóstico de laboratorio de infecciones gastrointestinales. México, D.F.: SSA, 1994; III-3:219-234. Documento interno de trabajo. 
nal; del grupo no humano, $57.6 \%$ se aisló de alimentos; $4.5 \%$, de agua, y $37.9 \%$, de muestras ambientales.

De las salmonelas aisladas de alimentos, $51 \%$ correspondió a alimentos preparados, $23 \%$ a cárnicos (productos derivados de la carne como: jamón, longaniza, chorizo, queso de puerco, etc.), $22 \%$ a carne (molida de res, pollo, pescado), $3 \%$ a lácteos y $1 \%$ a huevo (fresco y en polvo).

En el cuadro I se observa la distribución de los serotipos aislados de muestras humanas y no humanas. Durante estos años, se identificaron 199 serotipos diferentes, se encontraron algunos que no se habían aislado anteriormente. Como se puede observar, el serotipo más frecuente de muestras humanas fue $S$. Typhimurium, seguido de $S$. Enteritidis. En contraste, en muestras no humanas el serotipo más frecuente fue $S$. Derby, seguido de $S$. Anatum.

En la figura 1 se esquematiza el número de cepas aisladas en la República mexicana por origen de la muestra, humana y no humana, y enviadas a serotipificación al InDRE. Se puede observar un aumento gradual en el número de cepas tipificadas.

En la figura 2 se observa la variación de los cuatro serotipos más frecuentemente aislados de muestras humanas durante los 31 años considerados. Existe un cambio en la frecuencia de aislamiento de los diferentes serotipos en el periodo de 1990 a 1991. Estos cambios no correlacionan con los observados en muestras de origen no humano, pues la frecuencia de los serotipos es diferente entre los dos grupos.

\section{Discusión}

Los resultados encontrados en México son parecidos a algunos provenientes de otras partes del mundo: se han informado los cambios en la frecuencia de serotipos, y la mayoría coinciden en reportar a los serotipos $S$. Enteritidis y $S$. Typhimurium ${ }^{13-21}$ como los más frecuentes.

El principal serotipo aislado en muestras humanas fue $S$. Typhimurium encontrándose en diferentes porcentajes a través de los años, los cuales se deben a brotes de diarrea ocurridos entre la población, pero manteniendo un porcentaje constante entre 13 y $15 \%$ del total de serotipos. En cambio, $S$. Enteritidis se había estado aislando en un porcentaje muy bajo, con menos de $10 \%$ de frecuencia, pero a partir de 1991 se observó un incremento de cuatro veces en las muestras humanas. Este incremento, de menor magnitud, también se observa en las muestras no humanas, principalmente en las de alimentos, lo que sugiere que el incremento fue debido al consumo de alimentos contaminados por S. Enteritidis. Este hallazgo coincide con lo notificado por Rodrigue y colaboradores, quienes observan un incremento en el ámbito mundial de este serotipo. En Estados Unidos de América (EUA) este incremento se ha asociado al consumo de huevos contaminados, ${ }^{22}$ pero en México, en menos del $0.02 \%$, se ha aislado en huevos y la mayoría en aves $(0.6 \%)$; esto puede ser debido a que el mercado vende aves muy baratas importadas de EUA y a que existe una contaminación cruzada, además de que la población mexicana no consume alimentos preparados con huevos crudos como mayonesas caseras o huevos revueltos semicrudos.

De 1972 a 1989, S. Typhi ocupó el cuarto lugar de frecuencia; esto se debió a los brotes ocurridos en 1972 en la ciudad de México y estados circunvecinos, aumentando la proporción otros brotes ocurridos durante la década de los ochenta, y a partir de 1990 se observa un descenso en la frecuencia de aislamientos (1-2\%) el cual coincide con la entrada del cólera a México; durante ésta se implementaron medidas preventivas como hervir o clorar el agua antes de tomarla, lavarse las manos antes de comer y después de ir al baño. Esto impactó en una mejoría en las condiciones higiénicas de la población, previniendo no sólo los casos de cólera sino también los de tifoidea.

Otro serotipo importante en las muestras humanas fue $S$. Worthington que se aisló por primera vez en 1975 en una muestra de agua, manteniendo un porcentaje bajo de aislamientos (2\%). Sin embargo, a partir de 1989, se presentaron más aislamientos (10-20\%) debido a un brote intrahospitalario en el estado de Yucatán, el cual fue controlado una vez efectuadas las medidas higiénicas, y a partir de 1991 se siguió aislando en forma continua pero en baja proporción $(<1 \%)$.

En cuanto a las salmonelas aisladas de muestras no humanas, el serotipo principal fue $S$. Derby; el mayor número de aislamientos fue en alimentos, la mayoría analizados como parte del programa de regulación sanitaria. Fueron pocos los alimentos asociados a brotes por enfermedades transmitidas por alimentos (ETA), aunque los resultados nos hacen suponer que algunos de ellos fueron vehículos de ETA, ya que en muestras humanas se encontró este serotipo en $4.2 \%$; el segundo serotipo fue $S$. Anatum, con $8.5 \%$ de aislamientos. Es importante hacer notar que los serotipos aislados en muestras humanas tienen diferentes porcentajes que los que se aislaron en muestras no humanas y que algunos sólo se aislaron en estas últimas, lo cual puede deberse a la importación de carne y alimentos contaminados con salmonelas de diferentes serotipos y que éstas se mantuvieron en los alimentos sin causar aparentemente brotes en seres humanos. 


\section{Cuadro I}

\section{Distribución de serotipos de Salm on ella, según origen de la muestra. México, 1972-1999}

\begin{tabular}{|c|c|c|c|c|c|c|c|}
\hline Serotipo & Humano & No-humano & Total & Serotipo & Humano & No-humano & Total \\
\hline Typhimurium & 3225 & 578 & 3803 & Rubislaw & 11 & 12 & 23 \\
\hline Enteritidis & 2893 & 570 & 3463 & Paratyphi A & 19 & 3 & 22 \\
\hline Derby & 670 & 1176 & 1846 & Urbana & 4 & 16 & 20 \\
\hline Agona & 866 & 643 & 1509 & Choleraesuis & 11 & 8 & 19 \\
\hline Anatum & 608 & 748 & 1356 & Stanleyville & 11 & 5 & 16 \\
\hline Typhi & 1128 & 34 & 1162 & Reading & 6 & 7 & 13 \\
\hline N ewport & 865 & 235 & 1100 & Azteca & 5 & 7 & 12 \\
\hline Heidelberg & 623 & 211 & 834 & Cubana & 5 & 7 & 12 \\
\hline Infantis & 326 & 284 & 610 & Banana & 0 & 11 & 11 \\
\hline Worthington & 344 & 178 & 522 & Glostrup & 4 & 6 & 10 \\
\hline Oranienburg & 281 & 229 & 510 & Hato & 2 & 8 & 10 \\
\hline Hadar & 196 & 236 & 432 & Mendoza & 8 & 1 & 9 \\
\hline Muenchen & 305 & 116 & 421 & Chester & 2 & 6 & 8 \\
\hline Senftenberg & 129 & 238 & 367 & Gaminara & 4 & 4 & 8 \\
\hline Give & 108 & 244 & 352 & Irumu & 2 & 6 & 8 \\
\hline Saintpaul & 195 & 135 & 330 & Lille & 0 & 8 & 8 \\
\hline Panama & 229 & 87 & 316 & Pomona & 4 & 4 & 8 \\
\hline London & 79 & 178 & 257 & Stanley & 5 & 3 & 8 \\
\hline Mbandaka & 152 & 78 & 230 & Bergen & 0 & 7 & 7 \\
\hline Duesseldorf & 126 & 82 & 208 & Cannstatt & 4 & 3 & 7 \\
\hline Bovismorbificans & 74 & 125 & 199 & Colorado & 2 & 5 & 7 \\
\hline Poona & 156 & 43 & 199 & Liambu & 7 & 0 & 7 \\
\hline Cerro & 47 & 122 & 169 & Virchow & 2 & 5 & 7 \\
\hline Montevideo & 91 & 71 & 162 & Bradford & 6 & 0 & 6 \\
\hline W eltevreden & 122 & 37 & 159 & Luciana & 4 & 2 & 6 \\
\hline Adelaide & 57 & 97 & 154 & Menston & 6 & 0 & 6 \\
\hline O hio & 61 & 92 & 153 & Paratyphi C & 6 & 0 & 6 \\
\hline Braenderup & 118 & 32 & 150 & Vejle & 1 & 5 & 6 \\
\hline Minnesota & 53 & 47 & 100 & Carrau & 2 & 3 & 5 \\
\hline Brandenburg & 48 & 47 & 95 & Lindenburg & 3 & 2 & 5 \\
\hline Meleagridis & 29 & 59 & 88 & Shubra & 3 & 2 & 5 \\
\hline Bredeney & 40 & 46 & 86 & Elornrane & 0 & 4 & 4 \\
\hline Muenster & 48 & 37 & 85 & Manchester & 4 & 0 & 4 \\
\hline Livingstone & 21 & 59 & 80 & Tallahassee & 2 & 2 & 4 \\
\hline Havana & 34 & 36 & 70 & Weslaco & 0 & 4 & 4 \\
\hline Manhattan & 59 & 11 & 70 & W esthampton & 2 & 2 & 4 \\
\hline Arizona & 36 & 33 & 69 & Altona & 0 & 3 & 3 \\
\hline Blockley & 34 & 33 & 67 & Florida & 1 & 2 & 3 \\
\hline Indiana & 56 & 4 & 60 & Fyris & 1 & 2 & 3 \\
\hline Orion & 7 & 53 & 60 & Groenekan & 0 & 3 & 3 \\
\hline Amsterdam & 15 & 43 & 58 & Saphra & 3 & 0 & 3 \\
\hline Gallinarum & 18 & 38 & 56 & Sundsvall & 0 & 3 & 3 \\
\hline Sinstorf & 20 & 33 & 53 & Vancouver & 2 & 1 & 3 \\
\hline Shwarzengrund & 28 & 21 & 49 & Vilvoorde & 1 & 2 & 3 \\
\hline Tennessee & 17 & 27 & 44 & Abortusovis & 0 & 2 & 2 \\
\hline Lexington & 13 & 29 & 42 & Eppendorf & 0 & 2 & 2 \\
\hline Sandiego & 17 & 22 & 39 & Gabon & 0 & 2 & 2 \\
\hline Thompson & 29 & 10 & 39 & Godesberg & 1 & 1 & 2 \\
\hline Javiana & 27 & 11 & 38 & Hissar & 2 & 0 & 2 \\
\hline Bousso & 4 & 27 & 31 & $\| 1,6,7$ & 1 & 1 & 2 \\
\hline Kentucky & 11 & 20 & 31 & India & 0 & 2 & 2 \\
\hline$\overline{\text { Dublin }}$ & 26 & 4 & 30 & Isangi & 1 & 1 & 2 \\
\hline Paratyphi B & 20 & 10 & 30 & Kibusi & 1 & 1 & 2 \\
\hline Alachua & 18 & 8 & 26 & 91 serotipos & 768 & 540 & 1308 \\
\hline Blegdam & 24 & 1 & 25 & Rugosa & 78 & 147 & 225 \\
\hline
\end{tabular}




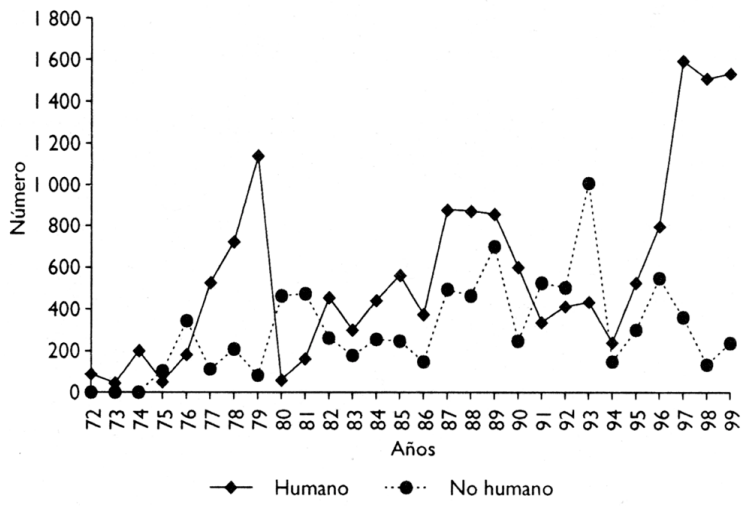

Figura 1. Número de cepas aisladas de Salmonella, SEgún ORIGen DE LA MUESTRA. Instituto de Diagnóstico y Referencia Epidemiológicos, México, 19721999

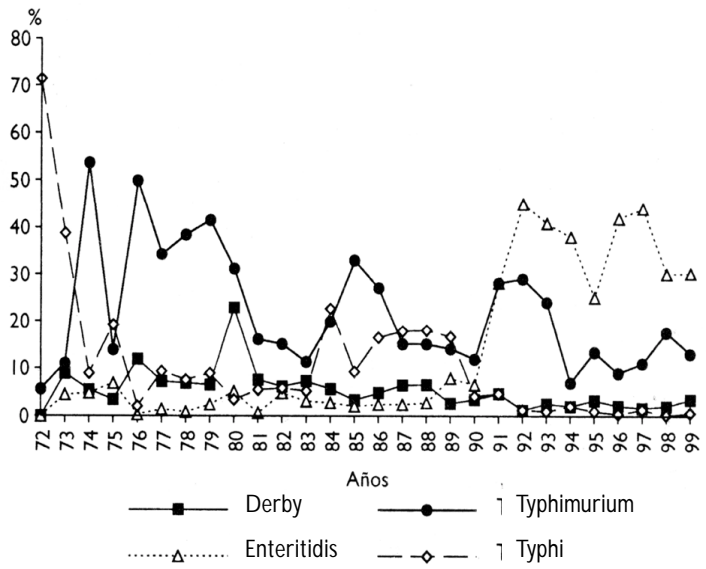

Figura 2.VAriación de los cuatro principales serotiPOS DE SALM O NeLLA AISLADOS DE HUMANOS. INSTITUTO DE Diagnóstico y Referencia Epidemiológicos, México, 1972-1999

Los aislamientos de Salmonella en alimentos preparados se pueden explicar por la manipulación de los alimentos así como por la contaminación propia de algunos de ellos, como las aves que al ser mal evisceradas contaminan la carne y en otros casos la contaminación cruzada de alimentos crudos con los preparados. Otros alimentos importantes fueron la carne cruda, los cárnicos o embutidos y los mariscos, los cuales presentan contaminación de origen y si no son debidamente preparados o almacenados dan lugar a una multiplicación de bacterias.
Se hicieron aislamientos también de animales, principalmente de aves y de ganado, lo que concuerda con lo notificado de que cerca de $80 \%$ de las aves están contaminadas y que éstas pueden estar infectando a otros animales, y de ellos pasar a los seres humanos, o bien, cuando se utilizan como desechos los restos de las aves (plumas, vísceras y picos) para preparar alimento para los propios animales lo que provoca un círculo vicioso.

El número de casos de salmonelosis anuales en México es muy alto comparado con el número de aislamientos por Salmonella, y esto es debido a que el diagnóstico se lleva a cabo en la mayoría de los casos sólo por clínica, por lo que es importante realizar el diagnóstico de laboratorio tanto en muestras clínicas como de alimentos y muestras ambientales, ya que esto servirá para conocer el comportamiento de los serotipos en diferentes fuentes y relacionar los serotipos encontrados en alimentos con los aislados de muestras clínicas.

En este estudio se observa que los serotipos más frecuentes aislados en México tanto en muestras humanas como no humanas son cinco $S$. Typhimurium, $S$. Enteritidis, $S$. Derby, $S$. Agona y $S$. Anatum; además de que los 194 serotipos restantes también son importantes clínicamente, aun los menos frecuentes, ya que tienen el mismo potencial de causar enfermedad que los que se aíslan con mayor frecuencia. Epidemiológicamente es importante conocer cuáles son los serotipos circulantes y los de nueva introducción para estar alerta en caso de que alguno de ellos presente un potencial de diseminación mayor que los demás.

\section{Agradecimientos}

A la doctora Dolores Correa Beltrán de la Dirección de Investigación y Desarrollo del InDRE, por el apoyo, la revisión y crítica constructiva al manuscrito. Al personal técnico de los laboratorios de bacteriología de la Red Nacional de Laboratorios de Salud Pública, de todas las instituciones del sector salud y de los laboratorios privados.

\section{Referencias}

1. Gutiérrez-Cogco L, González-Bonilla C, Giono-Cerezo S, Beltrán LG. Principales serotipos de Salmonella identificados en 10703 cepas en México entre 1982 y 1993. Rev Latinoam Microbiol 1994;36:221-226.

2. González-Bonilla C, Becerril P, Mendoza P, Bessudo D. Serotipos de salmonelas identificados en México entre 1974 y 1981. Bol O ficina Sanit Panam 1985; 99:34-39.

3. Secretaría de Salud. Boletín de Epidemiología. Sem 50. México, D.F.: Dirección General de Epidemiología, SSA, 1999. 
4. Dirección General de Epidemiología, Secretaría de Salud. Sistema único de información para la vigilancia epidemiológica. México, D.F.: SSA, 1994:92,187, 281.

5. Chalker RB, Blaser MJ. A review of human salmonellosis: III. Magnitude of Salmonella infection in the United States. Rev Infect D is 1988;10: 111-124.

6. Blaser MJ, N ewman LS. A review of human salmonellosis, I. Infective dose. Rev Infect D is 1992;4:1096-1106.

7. O rganización Mundial de la Salud. Control de la salmonelosis: importancia de la higiene veterinaria y de los productos de origen animal. Informe de un comité de expertos de la OMS. Ginebra: O MS, 1988;(Serie de Informes Técnicos núm. 774).

8. Instituto Panamericano de Protección de Alimentos y Zoonosis. Programa de Salud Pública Veterinaria. Buenos Aires: O rganización Mundial de la Salud, 1998:5-6.

9.Tauxe RV. Emerging foodborne diseases and envolving public health challenge. Emerg Infect D is 1997;(3)4:425-434.

10. Dirección General de Epidemiología, Secretaría de Salud. Sistema único de información para la vigilancia epidemiológica. México, D.F.: SSA, 1995;:l:89, 1995;Il:72, 1995;|ll:72, 1996;:l:120, 1996;ll:69, 1996;1:69, 1997:136, $232,424,1998: 172,266$ y 454.

11. 0 larte J,Varela G . Epidemiología de la salmonelosis en México. En:Van 0 ye $E$, ed.The world problem of Salmonellosis. La Haya:W Junk Publishers, 1964;554-563.

12. Popof MY. Institute Pasteur/Elsevier. Suplement 1995 (no. 39) to the Kauffmann-W hite Scheme. Rev Microbiol 1996;147:765-769.

13. Echeita MA, Diez R, U sera MA. Distribution of Salmonella spp. serotypes isolated in Spain during a 4-year period (1993-1996). Enferm Infecc Microbiol Clin 1999;17(1):9-14.
14. Echeita MA, Usera MA. Prevalence of Salmonella serotypes isolated in Spain from human and non human sources (1983-1987). Microbiologia 1989:5(2):95-103.

15. Usera MA, Cano R, Echeita A. Analysis of Salmonella sp. serotypes isolated in Spain in 1988-1992. Enferm Infecc Microbiol Clin 1995;13(3): 138-145.

16.Angeretti A, Martinetto $P, G$ affodio A M,A brardo T, Pugliese A. Isolation of Salmonella in the Piedmont from 1975 to 1978, with designation of rare strains and evaluation of the frequency of the serotypes. $G$ BatteriolVirol Immunol 1978;71(7-12):129-138.

17. Le Minor L, Le Minor S. O rigin and frequency of the serotypes of Salmonella isolated in France and received in the French $\mathrm{N}$ ational Center during the years 1977-1979. Rev Epidemiol Sante Publique 1981;29(1): 45-55.

18. Le Minor L, Le Minor S, Grimont PA. Q uadrennial report of the $\mathrm{N}$ ational Salmonella Center on the origin and distribution by serotypes of strains isolated in continental France during the years 1980 to 1983 . Rev Epidemiol Sante Publique 1985;33(1):13-21.

19. Eiguer T, Butta N , Picandet A M. Serotypes of Salmonella in the Republic of Argentina isolated from human and non-human sources 1979-1981. Rev Argent Microbiol 1983;15(1):25-31.

20. Khakhria R, W oodward D, Johnson W M, Poppe C. Salmonella isolated from humans, animals and other sources in Canada, 1983-92. Epidemiol Infect 1997;119(1):15-23.

21. C abrera-0 rtega R, Ramírez-Alvarez MM, Bravo-Fariñas L, G arcía-Rodríguez B, Fernández-A breuA.Serotipaje de los microorganismos pertenecientes al género Salmonella. Enf Infect Microbiol 1998;18(4):150-152. 22. Rodrigue DC, Tauxe RV, Rowe B. International increase in Salmonella enteritidis:A new pandemic? Epidemiol Infect 1990;105:21-27. 